\title{
Características epidemiológicas da paralisia cerebral em crianças e adolescentes em uma capital do nordeste brasileiro
}

\author{
Epidemiological characteristics of cerebral palsy in children and adolescents in a Brazilian \\ northeast capital
}

Características epidemiológicas de la parálisis cerebral en niños y adolescentes de una ciudad del Nordeste de Brasil

Marcus Valerius da Silva Peixoto', Andrezza Marques Duque², Susana de Carvalho³, Társilla Pereira Gonçalves ${ }^{4}$, Ana Paula de Souza Novais ${ }^{5}$, Marco Antônio Prado Nunes ${ }^{6}$

RESUMO|Oobjetivo desteestudo foi descrevera prevalência de paralisia cerebral entre crianças e adolescentes, seus subtipos, as possíveis comorbidades e as características socioeconômicas das famílias. Foi realizado um estudo epidemiológico do tipo transversal a partir de um inquérito de base populacional sobre a paralisia cerebral em crianças e adolescentes na cidade de Aracaju (SE), Brasil. O estudo obteve informações sobre 240 crianças e adolescentes com paralisia cerebral a partir das respostas a um questionário feitas por seus responsáveis. Foi encontrada a prevalência de período de 1,37 em cada mil. Alguns bairros possuem prevalência de três a quatro vezes maior, revelando que a taxa de prevalência total não é um indicador homogêneo. A maioria dos participantes foi do sexo masculino (56,25\%), de raça/cor declarada como parda ou preta (67,50\%), sendo que a média de idade foi de 8,56 anos. A paralisia cerebral de tipo espástica bilateral foi a mais frequente $(45,42 \%)$ e a comorbidade referida na maioria dos casos foi a epilepsia (48,33\%). A renda familiar mensal correspondia a \$252,87 dólares. O estudo revelou que as crianças e adolescentes com paralisia cerebral são, em grande parte, pertencentes a minorias sociais, de raça/cor parda ou preta, e suas famílias vivem na linha da extrema pobreza.

Descritores | Paralisia Cerebral; Epidemiologia; Determinantes Sociais.

\begin{abstract}
IThis study aimed to describe the prevalence of cerebral palsy among children and adolescents, its subtypes, associated comorbidities, and socioeconomic characteristics of families. This is a cross-sectional epidemiological study conducted from a populationbased survey on cerebral palsy among children and adolescents in the city of Aracaju, Sergipe, Brazil. Results: Based on guardians' answers to the proposed questionnaire, we obtained data on 240 children and adolescents with cerebral palsy in Aracaju. The period prevalence of cerebral palsy was 1.37 per 1,000 people. Some neighborhoods showed a prevalence three to four times higher than that found to the overall city, indicating that the total prevalence is not a homogeneous indicator. Most participants were male (56.25\%), self-declared as mixed-race or Black (67.50\%), and aged 8.56 years on average. Bilateral spastic was the most prevalent cerebral palsy subtype (45.42\%), and epilepsy was the most common comorbidity (48.33\%) along with intellectual disability. Household monthly income was \$252.87. Our results indicate that children and adolescents with cerebral palsy are mostly from minoritized groups, Black or mixedrace, and live in extreme poverty.
\end{abstract}

Keywords I Cerebral Palsy; Epidemiology; Social Determinants.

\footnotetext{
'Universidade Federal de Sergipe (UFS) - São Cristóvão (SE), Brasil. E-mail: peixotovalerius@gmail.com. Orcid: 0000-0001-9047-5875 2Universidade Federal de Sergipe (UFS) - Lagarto (SE), Brasil. E-mail: andrezza.duque@yahoo.com.br. Orcid: 0000-0002-6529-1887 3 Universidade Federal de Sergipe (UFS) - São Cristóvão (SE), Brasil. E-mail: susana_carvalho@uol.com.br. Orcid: 0000-0001-8708-3205 ${ }^{4}$ Universidade Federal de Sergipe (UFS) - São Cristóvão (SE), Brasil. E-mail: goncalves_tarsilla@hotmail.com. Orcid: 0000-0002-0027-9369 5Universidade Federal de Sergipe (UFS) - São Cristóvão (SE), Brasil. E-mail: aninhanovais123@gmail.com. Orcid: 0000-0002-4567-0783 GUniversidade Federal de Sergipe (UFS) - São Cristóvão (SE), Brasil. E-mail: manpn@ig.com.br. Orcid: 0000-0002-5416-4954
} 
RESUMEN I El objetivo de este estudio fue describir la prevalencia de parálisis cerebral en niños y adolescentes, sus subtipos, las posibles comorbilidades y las características socioeconómicas de las familias. Se realizó un estudio epidemiológico transversal a partir de una encuesta poblacional sobre parálisis cerebral en niños y adolescentes de la ciudad de Aracaju, Brasil. El estudio obtuvo información de 240 niños y adolescentes con parálisis cerebral con base en las respuestas de sus padres a un cuestionario. Se encontró la prevalencia de período de 1,37 por mil. Algunos barrios tienen una prevalencia de tres a cuatro veces mayor, lo que revela que la tasa de prevalencia total no es un indicador homogéneo.
La mayoría de los participantes eran varones (56,25\%), de raza/ color declarado parda o negra (67,50\%), con un promedio de edad de 8,56 años. La parálisis cerebral espástica bilateral fue la más frecuente (45,42\%), y la comorbilidad reportada en la mayoría de los casos fue la epilepsia (48,33\%). El ingreso familiar mensual correspondió a 252,87 dólares. El estudio reveló que los niños y adolescentes con parálisis cerebral son mayoritariamente de minorías sociales, raza/color parda o negra, y sus familias viven en extrema pobreza.

Palabras clave | Parálisis Cerebral; Epidemiología; Determinantes Sociales.

\section{INTRODUÇÃO}

A paralisia cerebral (PC) é a principal causa de incapacidade física na infância. As alterações estruturais e funcionais decorrentes da PC podem causar um grande impacto emocional, financeiro e social aos indivíduos, às famílias e às comunidades onde ela se apresenta, uma vez que ao longo da vida dos acometidos existem custos consideráveis em razão da necessidade de assistência à saúde ser mais frequente, dos cuidados com reabilitação e educação e das intervenções sociais. Crianças e jovens com PC têm condições de saúde significativamente piores $^{1}$ e soma-se a isso o fato de $\mathrm{o}$ atendimento às necessidades de pessoas com $\mathrm{PC}$ em locais vulneráveis e com poucos recursos ser um grande desafio ${ }^{2}$.

De acordo com uma revisão sistemática da literatura internacional, a prevalência da PC é de 2,11 a cada mil nascidos vivos e os fatores de risco mais conhecidos são: anormalidades placentárias, malformações congênitas, baixo peso ao nascer, aspiração de mecônio, cesariana de emergência, asfixia durante o parto, infecções e convulsões neonatais, síndrome do desconforto respiratório e hipoglicemia ${ }^{3,4}$.

Não foram encontrados estudos epidemiológicos que apontem a prevalência da PC no Brasil, no entanto, existe um grande empenho de pesquisadores no âmbito internacional para a produção de informações que descrevam a prevalência, as tendências, os tipo, as causas, as comorbidades, a funcionalidade e as associações da doença com as condições socioeconômicas dos indivíduos acometidos. O conhecimento das características da PC é essencial para planejar, executar e avaliar políticas e programas de prevenção e tratamento.
O uso de sistemas de informação e registros da PC em sistemas de vigilância é comum em países desenvolvidos e oferece dados para diferentes abordagens epidemiológicas e para o monitoramento da doença ${ }^{5-9}$. Em função da ausência de registros, como é o caso do Brasil, pesquisadores de países de baixa renda estão desenvolvendo estudos populacionais com informações relevantes para o meio científico e para suas localidades.

Dessa forma, este artigo tem como objetivo descrever a prevalência da PC entre crianças e adolescentes, seus subtipos, as comorbidades e as características socioeconômicas das famílias com base nos dados de uma capital do nordeste brasileiro.

\section{METODOLOGIA}

Foi realizado um estudo epidemiológico do tipo transversal a partir de um inquérito de morbidade, aplicado na atenção primária à saúde, sobre a $\mathrm{PC}$ em crianças e adolescentes na cidade de Aracaju (SE), Brasil.

\section{Área do estudo}

O estudo foi desenvolvido em Aracaju, capital do estado de Sergipe que ocupa uma área de $181,90 \mathrm{~km}^{2}$. De acordo com dados do Instituto Brasileiro de Geografia e Estatística $(\mathrm{IBGE})^{10}$, a cidade possui uma população estimada de 623.766 habitantes e apresenta densidade demográfica de $3.140,70 \mathrm{hab} / \mathrm{km}^{2}$. Aracaju tem 39 bairros e nenhuma área caracterizada como rural.

A cidade possui $93 \%$ de cobertura dos serviços de atenção primária à saúde para a população, com 44 serviços de saúde e 144 equipes de atenção primária. 


\section{Produção de dados}

A coleta de dados aconteceu entre junho de 2016 e dezembro de 2017 e foi conduzida por meio de uma busca ativa a partir de informantes-chave membros das equipes de atenção primária de todas unidades de saúde da cidade, as quais receberam um treinamento sobre a doença ministrada voluntariamente pela equipe de pesquisa visando qualificar a captação de informações sobre as crianças e adolescentes com PC. A pesquisa também foi realizada no único serviço público de atenção secundária e de referência para pessoas com PC, visando alcançar o maior número de participantes possível. A equipe de pesquisa coletou os dados nas residências das pessoas acometidas pela PC utilizando um questionário estruturado desenvolvido pelos pesquisadores.

Foram incluídas crianças e adolescentes com idade entre 0 e 18 anos que têm paralisia cerebral e que possuíam o diagnóstico médico no momento da coleta, registrado como G80.0-G80.9 na Classificação Estatística Internacional de Doenças e Problemas Relacionados com a Saúde (CID). Foram excluídos os casos de usuários do serviço de reabilitação de Aracaju que não residiam no território do município.

As variáveis observadas foram: sexo, idade, raça/cor, naturalidade, causas referidas, momento presumido do acometimento, local do parto, tipo de parto, idade gestacional, peso ao nascer, necessidade de internação após o nascimento, zona de residência, renda familiar, escolaridade da responsável, situação de trabalho da responsável e recebimento de benefício da previdência social.

Foi proposto o cálculo de prevalência de período para $\mathrm{PC}^{11}$ e, dessa forma, utilizou-se o denominador de mil crianças e adolescentes residentes em cada bairro. As informações sobre os residentes são provenientes do $\mathrm{IBGE}^{10}$.

Os dados foram submetidos a uma análise descritiva com medidas de tendência central e dispersão, também foram verificadas as frequências absolutas e relativas. Foi utilizado o software R ( $R$ Foundation for Statistical Computing, 2020), sendo que as funções do $R$-studio foram empregadas para a análise realizada, o que não demandou pacote estatístico específico.

Aplicou-se a técnica de análise exploratória de dados espaciais (Aede) para descrever a distribuição espacial da PC nos bairros utilizando o software $Q G I S$, versão 2.18.3 (Open Source Geospatial Foundation, 2016). A base cartográfica da cidade de Aracaju foi fornecida pelo IBGE. A projeção cartográfica corresponde ao Sistema de Referência Geocêntrico para as Américas (SIRGAS2000).

\section{RESULTADOS}

O estudo obteve informações sobre 240 crianças e adolescentes com PC na cidade de Aracaju a partir das respostas ao questionário proposto feitas por seus responsáveis. Considerando a população informada pelo censo demográfico brasileiro como 174.699 residentes com a mesma faixa etária, foi encontrada a prevalência de período de indivíduos com PC de 1,37 a cada mil habitantes. Entretanto, foram identificadas diferenças nas prevalências entre os bairros da cidade, que chegou até 4 a cada mil. A Figura 1 mostra os mapas coropléticos com a distribuição da PC por bairros, de acordo com a taxa de prevalência bruta a cada mil habitantes (1A) e a taxa de prevalência suavizada pelo modelo bayesiano empírico (1B). A exploração visual dos mapas mostra que existe maior prevalência na zona norte da cidade - região periférica - e menor prevalência na região central.

A maioria dos participantes foi do sexo masculino, de raça/cor declarada como parda ou preta, nascida em Aracaju e na faixa etária entre 0 e 4 anos (Tabela 1 ). A média de idade foi de 8,56 anos ( $\mathrm{DP}=5,76)$.

A PC do tipo espástica bilateral foi a mais frequente, entretanto, é importante destacar que mais de 30\% dos respondentes desconhecem o tipo de PC por conta de informações insuficientes nos diagnósticos. Nesses casos, os relatórios médicos indicaram o diagnóstico exclusivamente pelo CID G80.0. A comorbidade referida na maioria dos casos foi a epilepsia, seguida da deficiência intelectual. $\mathrm{O}$ conjunto de causas mais comuns para a $\mathrm{PC}$ referidas pelos responsáveis corresponde às complicações clínicas durante o parto e o momento da lesão mais relatado foi o perinatal (Tabela 2).

Foi referido pela maioria dos informantes que os partos ocorreram no ambiente hospitalar, com o tipo de parto vaginal sendo o mais recorrente, assim como o nascimento dos bebês a termo e com peso ao nascer acima de $2.500 \mathrm{~g}$. De acordo com os questionários, a maioria das crianças precisou ser internada logo após o nascimento (Tabela 3 ). 

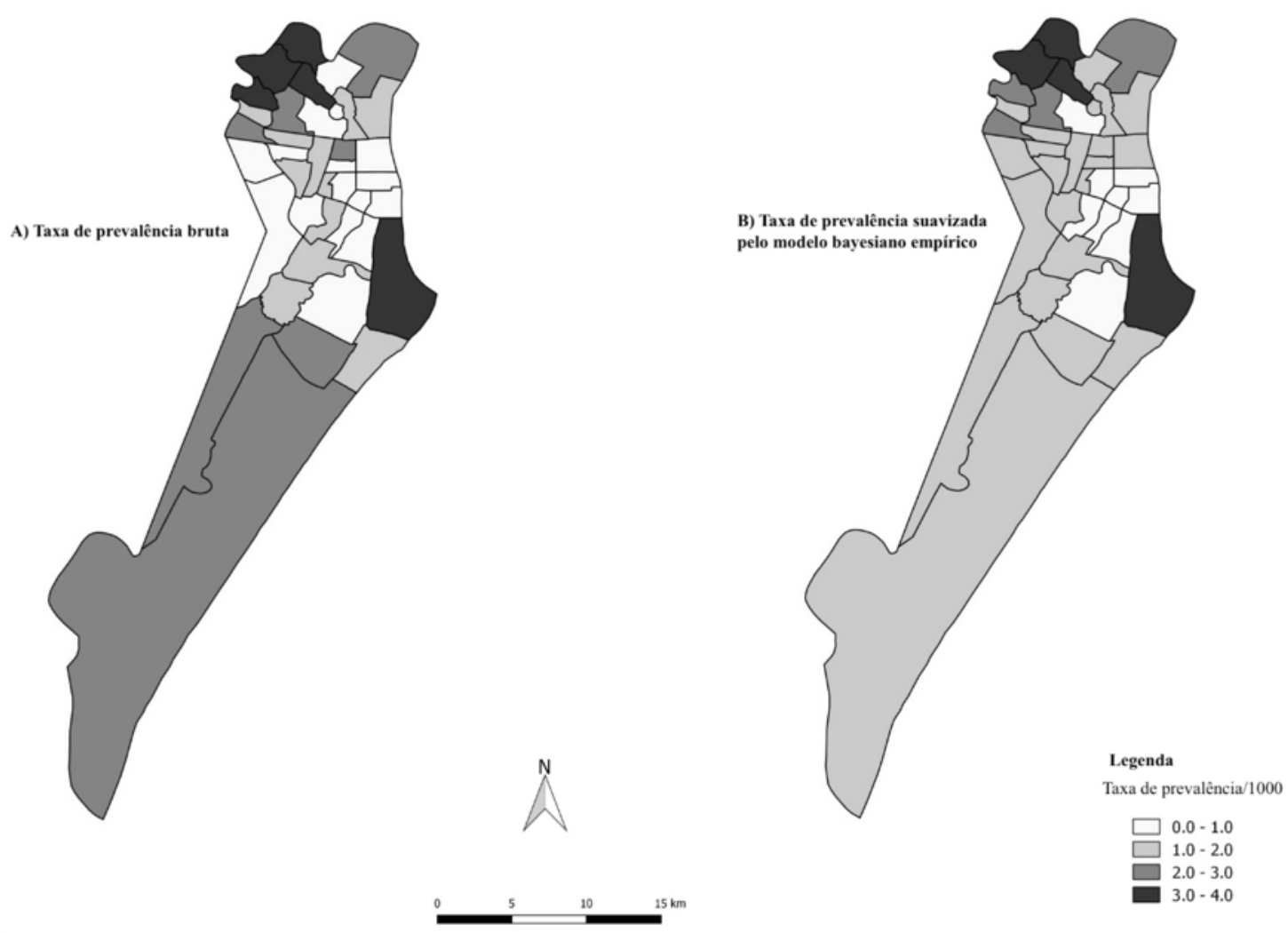

Figura 1. Mapas da distribuição espacial da prevalência de paralisia cerebral nos bairros de Aracaju (SE), Brasil, 2017

Tabela 1. Características demográficas das crianças e adolescentes com paralisia cerebral

$\begin{array}{lrr}\text { Variáveis } & \text { n } & \\ \text { Sexo } & 135 & 56,25 \\ \text { Masculino } & 105 & 43,75 \\ \text { Feminino } & & \\ \text { Raça/cor } & 16 & 6,67 \\ \text { Amarela } & 61 & 25,42 \\ \text { Branca } & 1 & 0,42 \\ \text { Indígena } & 162 & 67,50 \\ \text { Parda/Preta } & & \\ \text { Naturalidade } & 218 & 90,83 \\ \text { Aracaju } & 18 & 7,50 \\ \text { Outras cidades de Sergipe } & 4 & 1,67 \\ \text { Outros estados } & & \\ \text { Faixa etária } & 71 & 29,58 \\ \text { 0-4 anos } & 49 & 20,42 \\ \text { 5-8 anos } & 49 & 20,42 \\ \text { 9-12 anos } & 46 & 19,17 \\ \text { 13-16 anos } & 25 & 10,42 \\ \text { 17-18 anos } & 240 & 100 \\ \text { Total } & & \end{array}$

Tabela 2. Descrição dos subtipos de paralisia cerebral, das comorbidades, causas referidas e do momento presumido da lesão

\begin{tabular}{|c|c|c|}
\hline Variáveis & $\mathrm{n}$ & $\%$ \\
\hline \multicolumn{3}{|l|}{ Tipo de paralisia cerebral } \\
\hline Espástica bilateral & 109 & 45,42 \\
\hline Espástica unilateral & 46 & 19,17 \\
\hline Discinética & 5 & 2,08 \\
\hline Atáxica & 7 & 2,92 \\
\hline Não conhecido & 73 & 30,42 \\
\hline \multicolumn{3}{|l|}{ Comorbidades referidas* } \\
\hline Deficiência visual & 72 & 30,00 \\
\hline Deficiência auditiva & 22 & 9,17 \\
\hline Deficiência intelectual & 81 & 33,75 \\
\hline Distúrbios de comportamento & 51 & 21,25 \\
\hline Epilepsia & 116 & 48,33 \\
\hline \multicolumn{3}{|l|}{ Causa referida } \\
\hline Doenças congênitas & 64 & 26,67 \\
\hline Complicações na gestação & 34 & 14,17 \\
\hline Complicações clínicas no parto & 67 & 27,92 \\
\hline Falta de assistência no parto & 16 & 6,67 \\
\hline Doenças ou traumas da infância & 30 & 12,50 \\
\hline Não conhecido & 29 & 12,08 \\
\hline
\end{tabular}


Tabela 2. Continuação

$\begin{array}{lrr}\text { Variáveis } & \mathrm{n} & \% \\ \text { Momento presumido do acometimento } & & \\ \text { Pré-natal } & 81 & 33,75 \\ \text { Perinatal } & 102 & 42,50 \\ \text { Pós-natal } & 33 & 13,75 \\ \text { Não conhecido } & 24 & 10,00 \\ \text { Total } & 240 & 100\end{array}$

*Os participantes puderam referir mais de uma comorbidade.

Tabela 3. Características do nascimento de crianças e adolescentes com paralisia cerebral

\begin{tabular}{|c|c|c|}
\hline Variáveis & $\mathbf{n}$ & $\%$ \\
\hline \multicolumn{3}{|l|}{ Local do parto } \\
\hline Hospital & 230 & 95,83 \\
\hline Residência & 1 & 0,41 \\
\hline Não conhecido & 9 & 3,75 \\
\hline \multicolumn{3}{|l|}{ Tipo de parto } \\
\hline Vaginal & 123 & 51,25 \\
\hline Cesárea & 108 & 45,00 \\
\hline Não conhecido & 9 & 3,75 \\
\hline \multicolumn{3}{|l|}{ Idade gestacional } \\
\hline Pré-termo & 74 & 30,83 \\
\hline Termo & 143 & 59,58 \\
\hline Pós-termo & 11 & 4,58 \\
\hline Não conhecido & 23 & 9,58 \\
\hline \multicolumn{3}{|l|}{ Peso ao nascer } \\
\hline$<1.000 \mathrm{~g}$ & 4 & 1,67 \\
\hline$\geq 1.000 \mathrm{~g} \mathrm{e}<1.500 \mathrm{~g}$ & 12 & 5,00 \\
\hline$\geq 1.500 \mathrm{~g} \mathrm{e}<2.500 \mathrm{~g}$ & 42 & 17,50 \\
\hline$\geq 2.500 \mathrm{~g}$ & 117 & 48,75 \\
\hline Não conhecido & 65 & 27,08 \\
\hline \multicolumn{3}{|c|}{ Internação após nascimento } \\
\hline Sim & 125 & 52,58 \\
\hline Não & 96 & 40,00 \\
\hline Não conhecido & 19 & 7,92 \\
\hline Total & 240 & 100 \\
\hline
\end{tabular}

Os dados de renda familiar foram coletados de acordo com o valor do salário mínimo estabelecido no Brasil, que no ano de 2016 correspondia a $\$ 252,87$ dólares. Os familiares que convivem na mesma residência com as crianças e adolescentes analisadas somam um total de 987 pessoas, uma média de 4,11 pessoas por domicílio. A maioria das famílias possuía apenas um salário mínimo por mês como renda. Foram mais frequentes as mães ou responsáveis que tinham entre nove e doze anos de estudo, o que correspondente à escolaridade de nível médio; a atividade de trabalho preponderante foi a de dona de casa, o que inclui os cuidados dispensados para a pessoa com PC. O benefício da previdência social foi concedido para a maioria dos participantes, segundo os critérios do governo brasileiro, a renda familiar deve ser de $1 / 4$ de salário mínimo por mês para que as famílias estejam aptas a receber o benefício (Tabela 4).

\begin{tabular}{|c|c|c|}
\hline Variáveis & $\mathbf{n}$ & $\%$ \\
\hline \multicolumn{3}{|c|}{ Renda familiar em salários mínimos } \\
\hline Até 1 salário mínimo & 140 & 58,33 \\
\hline 2 & 55 & 22,92 \\
\hline 3 & 24 & 10,00 \\
\hline 4 & 11 & 4,58 \\
\hline 5 salários mínimos ou mais & 10 & 4,17 \\
\hline \multicolumn{3}{|c|}{ Escolaridade do responsável em anos de estudo } \\
\hline $0-4$ & 30 & 12,5 \\
\hline $5-8$ & 62 & 25,83 \\
\hline $9-12$ & 94 & 39,17 \\
\hline $13-18$ & 54 & 22,5 \\
\hline \multicolumn{3}{|c|}{ Situação de trabalho da mãe ou responsável } \\
\hline Desempregada & 40 & 16,67 \\
\hline Trabalho informal & 8 & 3,33 \\
\hline Trabalho formal & 17 & 7,08 \\
\hline Funcionária pública & 50 & 20,83 \\
\hline Autônoma & 25 & 10,42 \\
\hline Dona de casa & 100 & 41,67 \\
\hline \multicolumn{3}{|l|}{ Benefício da previdência social } \\
\hline Sim & 172 & 71,67 \\
\hline Não & 68 & 28,33 \\
\hline Total & 240 & 100 \\
\hline
\end{tabular}

\section{DISCUSSÃO}

A prevalência de $\mathrm{PC}$ na população estudada foi mais baixa que em estudos realizados em países de baixa renda, como Uganda; de média renda, como China e Turquia, ou países de alta renda da Europa ${ }^{9,12-14}$. As diferenças de prevalência dentro de Aracaju são relevantes, visto que alguns bairros na região norte - periferia da cidade - possuem prevalência de três a quatro vezes maior, revelando que a taxa de prevalência total não é um indicador homogêneo e que esta incapacidade física na infância pode ter relações com as iniquidades sociais.

Um estudo realizado no norte da Inglaterra propôs relatar o agrupamento espaço-temporal entre os casos de $\mathrm{PC}$ e encontrou resultados consistentes que associam o aparecimento da doença com exposições a infecções maternas que ocorrem durante o período perinatal. Os agrupamentos foram encontrados em áreas mais densamente povoadas ${ }^{15}$.

Uma revisão sistemática da literatura que analisou a relação entre status socioeconômico e risco de PC revelou associações significantes, mesmo após o controle do peso ao nascer e a idade gestacional como variáveis 
confundidoras $^{16}$. Fatores de risco conhecidos para a PC, como baixo peso, infecções maternas, malformações congênitas e dificuldades de assistência pré-natal, são preveníveis em grande parte dos casos e podem ter seus desfechos indesejados atenuados se forem produzidas informações espaciais como as que foram abordadas neste estudo ${ }^{15,17}$.

Sugere-se que outros estudos de tendência sejam realizados considerando este como base objetivando compreender melhor o que origina essa diferença. Acredita-se que a baixa prevalência não foi determinada por possíveis limitações no rastreamento dos casos, porque a cobertura da atenção primária de Aracaju é feita por território delimitado e bem conhecido pelas equipes de atenção primária que foram as informantes-chave para acessar as famílias.

A maioria absoluta dos participantes é natural de Aracaju, e aparentemente o fenômeno de migração não está interferindo na prevalência local de PC. Entretanto, é possível que o subdiagnóstico de crianças com PC leve, bem como a mortalidade desse grupo, possa interferir nesse valor. Sugere-se, assim, que análises de mortalidade sejam feitas futuramente, uma vez que existe um predomínio da doença entre crianças de 0 a 12 anos, que compõem mais de $70 \%$ da população do estudo.

Foi identificada uma grande disparidade étnica entre os indivíduos acometidos, com maior ocorrência da $\mathrm{PC}$ em pardos e pretos. Outros estudos evidenciaram resultados compatíveis em outros contextos ${ }^{18}$. Uma coorte retrospectiva realizada na Califórnia (Estados Unidos) verificou que bebês negros tinham 29\% mais chances de ter PC do que os brancos ${ }^{19}$. Outro estudo que analisou três áreas dos Estados Unidos reforça que o risco de PC espástica foi mais de $50 \%$ maior para crianças negras ${ }^{20}$. Os estudos não explicam totalmente as disparidades raciais, e se levanta a hipótese do maior risco de baixo peso ao nascer em crianças negras, mas também pode ser considerada a maior vulnerabilidade socioeconômica da população negra, o que inclusive interfere no acesso aos serviços de saúde.

O tipo de PC espástica bilateral também foi observado como o mais frequente em outras pesquisas publicadas, uma realizada em Uganda (45\%) e outra realizada na Noruega $(49 \%)^{21,22}$. Os trabalhos evidenciam, assim como a presente pesquisa, que as comorbidades mais comuns são epilepsia e dificuldades de aprendizagem. Uma revisão sistemática da literatura sobre a PC em países de baixa renda mostra taxas aumentadas de quadriplegia espástica para estudos realizados em ambulatórios ${ }^{23}$.
Foi referido pelos informantes que a maioria dos casos resulta de complicações durante o parto, com o presumido evento de acometimento ocorrendo no momento perinatal. Esse dado alerta para prováveis problemas nas condições de parto e nascimento, por exemplo, na assistência à gestante e ocorrência de asfixia ao nascer. Um estudo realizado na Turquia relatou que os fatores pré-natais influenciadores da PC foram observados com maior frequência nos grupos com alto nível socioeconômico, enquanto os fatores perinatais foram mais encontrados em indivíduos com baixo nível socioeconômico ${ }^{14}$.

O maior volume de crianças e adolescentes nascidos a termo e com peso acima de $2.500 \mathrm{~g}$ deve ser interpretado com cautela, uma vez que a prematuridade e o baixo peso ao nascer são internacionalmente reconhecidos como os principais fatores de risco da $\mathrm{PC}^{2,4}$. Um estudo considerou o baixo peso ao nascer e a prematuridade como mediadores da associação dos fatores socioeconômicos com a $\mathrm{PC}^{24}$. A frequência de casos relatados com idade gestacional e peso típico foi considerável e pode ser mais um elemento que indica a ocorrência de eventos perinatais. Uma pesquisa realizada na China e outra realizada na Coreia encontraram dados semelhantes utilizando a contribuição majoritária de crianças, de $2.500 \mathrm{~g}$, nascidas com PC $\mathrm{PC}^{13,25}$. Nesse sentido, levanta-se a hipótese de que as lesões ocorridas durante o parto contribuem fortemente para este perfil.

Os dados de renda familiar permitem afirmar que grande parte dessa população (aproximadamente $60 \%$ ) possui renda per capita de dois dólares por dia, compatível com a situação de pobreza extrema, segundo o Banco Mundial ${ }^{26}$. Uma pesquisa realizada em Taiwan encontrou uma forte relação da renda familiar com a maior prevalência da $\mathrm{PC}^{27}$. Outros estudos em países de alta renda relatam a associação entre o gradiente socioeconômico e a $\mathrm{PC}^{16,17,28}$. As evidências levantadas para essa população, como mostram os demais estudos, corroboram que a prevenção da PC deve passar, sobretudo, pelo enfrentamento da pobreza e das desigualdades sociais. A PC é a principal causa de incapacidade física na infância e também uma das responsáveis pelos maiores custos para as famílias e para os sistemas de saúde ${ }^{29}$. Assim, agir sobre a pobreza e as diferenças sociais é também agir sobre os determinantes da saúde e sobre uma das causas da doença.

Um estudo baseado em duas coortes na Dinamarca e na Noruega verificou uma forte tendência de diminuição do risco de PC conforme o nível de educação da mãe e do pai subiam, visto que esse fator pode influenciar 
potencialmente no estilo de vida e em comportamentos de saúde modificáveis ${ }^{30}$. A maioria das mães ou responsáveis que participaram do estudo em Aracaju possui escolaridade equivalente ao ensino médio em anos de estudo, o que pressupõe que tenham competência para decidir sobre a busca do cuidado em saúde, bem como sobre os comportamentos que não prejudiquem a saúde das gestantes. Entretanto, os demais dados socioeconômicos sugerem menores oportunidades sociais, o que interfere na prevalência da PC.

As mães ou responsáveis desempregadas ou donas de casa somam uma grande proporção neste estudo. Esse dado revela o quanto as mulheres e famílias ficam economicamente vulneráveis em função da PC. As dificuldades funcionais provocadas pela deficiência física e pelas comorbidades geram uma excessiva carga de trabalhos domésticos para as cuidadoras e impedem a busca por trabalho remunerado no meio profissional.

Esta pesquisa apresenta limitações que são inerentes ao desenho de estudo de tipo transversal, por exemplo, a possibilidade de viés de memória e as dificuldades para determinar estatisticamente como as variáveis interagem para o desfecho da PC. Entretanto, as dificuldades foram atenuadas pela qualidade de elaboração do questionário e pelo tamanho da amostra, uma vez que buscou-se acessar a maior quantidade possível de pessoas com $\mathrm{PC}$ residentes em Aracaju, e compreende-se que, apesar de os dados serem descritivos, podem sugerir relevantes associações.

As vantagens desta pesquisa se devem ao fato de que se trata de um estudo de base populacional que abrangeu todo o território da cidade e acessou não apenas as pessoas que frequentam ambulatórios de reabilitação. Além disso, o estudo fornece importantes informações para a formulação, implementação e avaliação de políticas públicas. Destaca-se também que é uma pesquisa que pode servir de base para comparação com os resultados de outros países e com séries históricas de outros locais do Brasil.

\section{CONCLUSÃO}

A PC na população estudada apresenta uma prevalência menor em relação a outros países. O subtipo mais comum foi a espástica bilateral, com epilepsia e deficiência intelectual como comorbidades mais presentes. As crianças e os adolescentes com PC são em grande parte de minorias sociais vulneráveis, de raça/cor parda ou preta, e suas famílias vivem na linha da extrema pobreza.
As informações produzidas neste estudo contribuem para o monitoramento e a comparação com futuras pesquisas no país e com dados de outros países.

\section{AGRADECIMENTOS}

Agradecemos à Secretaria Municipal de Saúde de Aracaju, ao Centro de Educação Permanente em Saúde, às equipes de saúde da família e em especial aos agentes comunitários de saúde pela parceria. Um agradecimento especial aos voluntários e voluntárias, mães, pais e responsáveis pela colaboração e à Universidade Federal de Sergipe.

\section{REFERÊNCIAS}

1. McCullough N, Parkes J, Kerr C, McDowell BC. The health of children and young people with cerebral palsy: A longitudinal, population-based study. Int J Nurs Stud. 2013;50(6):747-56. doi: 10.1016/j.ijnurstu.2011.01.011

2. Graham HK, Rosenbaum P, Paneth N, Dan B, Lin J-P, Damiano DL, et al. Cerebral palsy. Nat Rev Dis Primers. 2016;2:15082. doi: 10.1038/nrdp.2015.82

3. Pakula AT, Van Naarden Braun K, Yeargin-Allsopp M. Cerebral Palsy: classification and epidemiology. Phys Med Rehabil Clin N Am. 2009;20(3):425-52. doi: 10.1016/j.pmr.2009.06.001

4. Mclntyre S, Taitz D, Keogh J, Goldsmith S, Badawi N, Blair E. A systematic review of risk factors for cerebral palsy in children born at term in developed countries. Dev Med Child Neurol. 2013;55(6):499-508. doi: 10.1111/dmcn.12017

5. Dan B, Paneth N. Making sense of cerebral palsy prevalence in low-income countries. Lancet Glob Heal. 2017;5(12):e1174-5. doi: 10.1016/S2214-109X(17)30420-5

6. Himmelmann K, Uvebrant P. The panorama of cerebral palsy in Sweden part XII shows that patterns changed in the birth years 2007-2010. Acta Paediatr. 2018;107(3):462-8. doi: 10.1111/ apa.14147

7. Goldsmith S, Mclntyre S, Smithers-Sheedy H, Blair E, Cans C, Watson L, et al. An international survey of cerebral palsy registers and surveillance systems. Dev Med Child Neurol. 2016;58(Suppl 2):11-7. doi: 10.1111/dmcn.12999

8. Cans C, Surman G, McManus V, Coghlan D, Hensey O, Johnson A. Cerebral palsy registries. Semin Pediatr Neurol. 2004;11(1): 18-23. doi: 10.1016/j.spen.2004.01.004.

9. Sellier E, Platt MJ, Andersen GL, Krägeloh-Mann I, De La Cruz J, Cans C, et al. Decreasing prevalence in cerebral palsy: a multi-site European population-based study, 1980 to 2003. Dev Med Child Neurol. 2016;58(1):85-92. doi: 10.1111/dmcn.12865

10. Brasil. Instituto Brasileiro de Geografia e Estatística. Cidades e Estados, Aracaju-SE [Internet]. Brasília, DF: IBGE; [cited 2018 Dez 20]. Available from: https://www.ibge.gov.br/cidades-eestados/se/aracaju.html 
11. Van Naarden Braun K, Maenner MJ, Christensen D, Doernberg NS, Durkin MS, Kirby RS, et al. The role of migration and choice of denominator on the prevalence of cerebral palsy. Dev Med Child Neurol. 2013;55(6):520-6. doi: 10.1111/dmcn.12095

12. Kakooza-Mwesige A, Andrews C, Peterson S, Wabwire Mangen F, Eliasson AC, Forssberg H. Prevalence of cerebral palsy in Uganda: a population-based study. Lancet Glob Health. 2017:5(12):e1275-82. doi: 10.1016/S2214-109X(17)30374-1

13. Liu JM, Li S, Lin Q, Li Z. Prevalence of cerebral palsy in China. Int J Epdemiol. 1999;28(5). doi: 10.1093/ije/28.5.949

14. Serdaroğlu A, Cansu A, Özkan S, Tezcan S. Prevalence of cerebral palsy in Turkish children between the ages of 2 and 16 years. Dev Med Child Neurol. 2006;48(6):413-6. doi: 10.1017/ S0012162206000910

15. McNally RJQ, Colver AF. Space-time clustering analyses of occurrence of cerebral palsy in Northern England for births 1991 to 2003. Ann Epidemiol [Internet]. 2008;18(2):108-12. doi: 0.1016/j.annepidem.2007.07.104

16. Solaski M, Majnemer A, Oskoui M. Contribution of socioeconomic status on the prevalence of cerebral palsy: a systematic search and review. Dev Med Child Neurol. 2014;56(11):1043-51. doi: 10.1111/dmcn.12456

17. Durkin MS, Maenner MJ, Benedict RE, Van Naarden Braun K, Christensen D, Kirby RS, et al. The role of socio-economic status and perinatal factors in racial disparities in the risk of cerebral palsy. Dev Med Child Neurol. 2015;57(9):835-43. doi: $10.1111 / d m c n .12746$

18. Van Naarden Braun K, Doernberg N, Schieve L, Christensen D, Goodman A, Yeargin-Allsopp M. Birth prevalence of cerebral palsy: a population-based study. Pediatrics. 2016;137(1):e20152872. doi: 10.1542/peds.2015-2872

19. Wu YWW, Xing G, Fuentes-Afflick E, Danielson B, Smith LH, Gilbert WM. Racial, ethnic, and socioeconomic disparities in the prevalence of cerebral palsy. Pediatrics. 2011;127(3):e674-81. doi: 10.1542/peds.2010-1656

20. Yeargin-Allsopp M, Van Naarden Braun K, Doernberg NS, Benedict RE, Kirby RS, Durkin MS. Prevalence of cerebral palsy in 8-year-old children in three areas of the United States in 2002: a multisite collaboration. Pediatrics. 2008;121(3):547-54. doi: 10.1542/peds.2007-1270
21. Kakooza-Mwesige A, Forssberg H, Eliasson AC, Tumwine JK. Cerebral palsy in children in Kampala, Uganda: Clinical subtypes, motor function and co-morbidities. BMC Res Notes. 2015;8:166. doi: 10.1186/s13104-015-1125-9

22. Andersen GL, Irgens LM, Haagaas I, Skranes JS, Meberg AE, Vik T. Cerebral palsy in Norway: prevalence, subtypes and severity. Eur J Paediatr Neurol. 2008;12(1):4-13. doi: 10.1016/j. ejpn.2007.05.001

23. Gladstone M. A review of the incidence and prevalence, types and aetiology of childhood cerebral palsy in resourcepoor settings. Ann Trop Paediatr. 2010;30(3):181-96. doi: 10.1179/146532810×12786388978481

24. Sundrum R, Logan S, Wallace A, Spencer N. Cerebral palsy and socioeconomic status: a retrospective cohort study. Arch Dis Child. 2005;90(1):15-8. doi: 10.1136/adc.2002.018937

25. Yim SY, Yang CY, Park JH, Kim MY, Shin YB, Kang EY, et al. Korean Database of Cerebral Palsy: A Report on Characteristics of Cerebral Palsy in South Korea. Ann Rehabil Med. 2017;41(4): 638-49. doi: 10.5535/arm.2017.41.4.638

26. World Bank. Quase metade do mundo vive com menos de USD $\$ 5.50$ por dia. [Internet]. Washington, DC: World Bank; 2018 [cited 2018 Dec 20]. Available from: https://www.worldbank. org/pt/news/press-release/2018/10/17/nearly-half-the-worldlives-on-less-than-550-a-day-brazilian-portuguese

27. Tseng SH, Lee JY, Chou YL, Sheu ML, Lee YW. Association between socioeconomic status and cerebral palsy. PLoS One. 2018;13(1):e0191724. doi: 10.1371/journal.pone.0191724

28. Oskoui M, Messerlian C, Blair A, Gamache P, Shevell M. Variation in cerebral palsy profile by socio-economic status. Dev Med Child Neurol. 2016:58(2):160-6. doi: 10.1111/dmcn.12808

29. Shih STF, Tonmukayakul U, Imms C, Reddihough D, Graham HK, Cox L, et al. Economic evaluation and cost of interventions for cerebral palsy: a systematic review. Dev Med Child Neurol. 2018;60(6):543-58. doi: 10.1111/dmcn.13653

30. Forthun I, Strandberg-Larsen K, Wilcox AJ, Moster D, Petersen TG, Vik T, et al. Parental socioeconomic status and risk of cerebral palsy in the child: evidence from two Nordic population-based cohorts. Int J Epidemiol. 2018;47(4): 1298-306. doi: 10.1093/ije/dyy139 